\title{
A Novel Method of Optimization for Stochastic Control System
}

\author{
Yupeng Wen \\ School of Science, Jilin Institute of Chemical Technology, Jilin, China \\ Received: October 15, 2020. Revised: November 23, 2020. Accepted: December 10, 2020. \\ Published: December 14, 2020.
}

\begin{abstract}
Stochastic phenomena widely exist in the nature and real dynamic systems. The existence of random phenomena will make the system performance degrade greatly, and even cause instability. For the sake of improving the stability of stochastic control system, this paper proposed a novel method of optimization for stochastic control system by control model and max-plus algebraic algorithm. The simulation results indicate that the optimization method can effectively optimize the stochastic system. The input of the stochastic control system is stable to a certain extent, which weakens the random interference of the input signal in the external environment, thus improving the stability of the stochastic control system.
\end{abstract}

Keywords-stochastic control system, stability, predictive control, random interference

\section{INTRODUCTION}

$\mathrm{S}$ tochastic control system a dynamic system affected by random factors. It is usually described by stochastic differential equation or stochastic difference equation. From the perspective of control theory, the research on stochastic systems mainly includes the following aspects.

The mathematical model for the system is set up from the measured data, or the model structure of the system has been given, and the unknown parameters in the model can be estimated according to the measured data. Specifically, in terms of modeling and identification, white noise driven difference equation (ARMA process) is often used to model the measured random data. The estimation of the coefficient and order of ARMA process is called time series analysis. It has a set of mature methods, but the process that can be effectively handled by this method usually needs to have stationarity. However, the output process of feedback control system is not stable due to the control term, so the commonly used time series analysis method does not estimate the parameters of the feedback control system Applicable. ARMAX process is the most commonly used model for stochastic control system modeling. Its coefficient estimation is usually estimated by least squares, maximum likelihood or other algorithms derived from it. However, if the estimation converges to the true value, the system must be stimulated to a certain extent. However, how large the excitation is and how fast the convergence speed is, this has caused a lot of research. The order estimation of feedback control systems is an interesting problem. According to the measured data and system model, the system state or signal disturbed by noise is estimated. The control of stochastic system is constructed to minimize the given performance index.

Due to the actual systems in engineering technology, environmental ecology, social economy and other fields, there are generally random disturbances. Therefore, the study of stochastic systems is necessary for practical application, but it also brings many difficult theoretical issues. So for a long time, stochastic control systems have been widely concerned by researchers of various backgrounds.

Stochastic phenomena widely exist in the nature and real dynamic systems. The existence of random phenomena will make the system performance degrade greatly, and even cause instability. This paper proposed a novel method of optimization for stochastic control system by control model and genetic algorithm.

\section{PREDICTIVE CONTROL}

Predictive control (PC) has been successfully applied to industrial process control because of its strong robustness to the uncertainty of the controlled plant and the ability to overcome the influence of pure delay on the characteristics of closed-loop systems.

The structure of predictive control system is shown in Figure 1. 


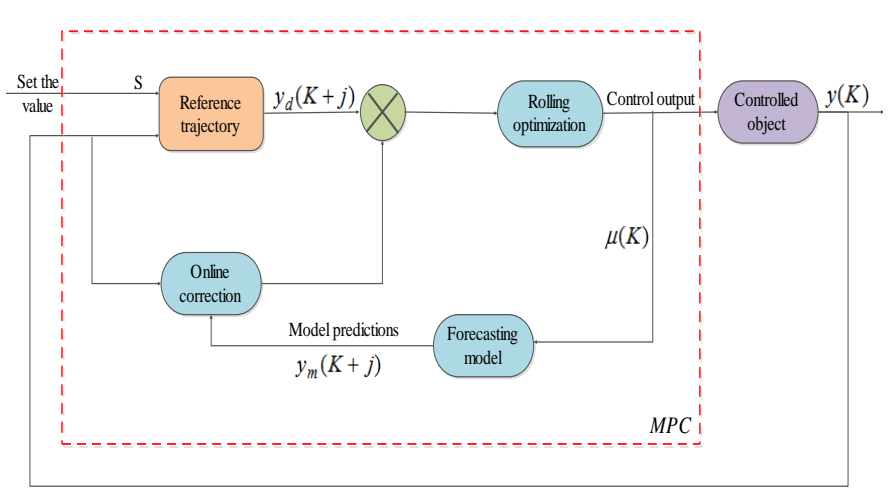

Figure 1. The structure of predictive control system

However, PC is mainly concentrated on stochastic control system, and a common industrial process contains complex nonlinear characteristics. Therefore, the research scope of PC has been extended from linear stochastic control system to nonlinear stochastic control system.

The input or output model of the control object can be described as

$$
y(k)=f(y(k-1), \cdots, y(k-m), u(k-1), \cdots, u(k-n))
$$

where $u(k), y(k)$ denote the input and output of the system respectively, $n$ and $m$ are the orders of the input and output respectively. $f(\cdot)$ is an unknown nonlinear continuous differentiable function with respect to $y(k-1), \cdots, y(k-m), u(k-1), \cdots, u(k-n)$, and meets following conditions.

A1) $f(\underbrace{0, \cdots, 0}_{n}, \underbrace{0,0, \cdots, 0}_{m})=0$

A2) $f(\cdot)$ is continuous differentiable with respect to $y(k-1), \cdots, y(k-m), \quad u(k-1), \cdots, u(k-n)$, and every partial derivative is bounded, suppose $0<\frac{\partial f}{\partial u(k-N)} \leq k_{\max }$, where $k_{\max }$ is a constant, $N$ is the prediction horizon.

The nonlinear system (1) satisfying the conditions A1) and A2) can be expressed by formula (2) as a time-varying linear system.

$$
\begin{aligned}
y(k) & =f(y(k-1), \cdots, y(k-m), u(k-1), \cdots, u(k-n)) \\
& =\sum_{i=1}^{m} \bar{a}_{i}(k) y(k-i)+\sum_{i=1}^{n} \bar{b}_{i}(k) u(k-i)
\end{aligned}
$$

In the equation,

$$
\begin{gathered}
\bar{a}_{i}(k)=\left.\frac{\partial f}{\partial y(k-i)}\right|_{\left(y(k-1), \cdots, y(k-i+1), \xi_{y}, 0, \cdots, 0\right)} \\
i=1, \cdots, m, \xi_{y} \in(0, \quad y(k-i))
\end{gathered}
$$

$$
\begin{array}{r}
\bar{b}_{i}(k)=\left.\frac{\partial f}{\partial u(k-i)}\right|_{\left(y(k-1), \cdots, y(k-n), u(k-1), \cdots, u(k-i+1), \xi_{u}, 0, \cdots, 0\right)} \\
i=1, \cdots, n, \xi_{u} \in(0, \quad u(k-i))
\end{array}
$$

The controlled object can be described with the time-varying model as formula (3).

$$
A\left(k, z^{-1}\right) \Delta y(k)=B\left(k, z^{-1}\right) \Delta u(k-1)
$$

Where $u(k)$ and $y(k)$ represent the input and output of the controlled object,

$$
\begin{aligned}
& A\left(k, z^{-1}\right)=1+a_{1}(k) z^{-1}+\cdots+a_{n_{a}}(k) z^{-n_{a}} \\
& B\left(k, z^{-1}\right)=b_{0}(k)+b_{1}(k) z^{-1}+\cdots+b_{n_{b}}(k) z^{-n_{b}}
\end{aligned}
$$

$n_{a}=m, n_{b}=n-1, \Delta=1-z^{-1}$ represent the difference operator, given the lag of the controlled object $d=1$, if $d>1$, only need to let the coefficients of preceding $d-1$ terms in the $B\left(k, z^{-1}\right)$ polynomial equal zero.

To make the output $y(k+j)$ of the controlled object tracking the reference sequence $y_{r}(k+j)(j=1,2, \cdots)$, let the performance indicator function as

$$
J=\sum_{j=1}^{N}\left(y(k+j)-y_{r}(k+j)\right)^{2}
$$

The vectorial form of the optimal prediction output can be obtained as formula (5) by introducing the Diophantine equation,

$$
\boldsymbol{Y}=\boldsymbol{G} \boldsymbol{U}+\boldsymbol{F} y(k)+\boldsymbol{H} \Delta u(k-1)
$$

Defining $\quad \boldsymbol{Y}_{r}=\left[y_{r}(k+1), \cdots, y_{r}(k+N)\right]^{\mathrm{T}}, \quad$ then the performance indicator function (4) can be written as formula (6)

$$
J=\left(\boldsymbol{Y}-\boldsymbol{Y}_{r}\right)^{\mathrm{T}}\left(\boldsymbol{Y}-\boldsymbol{Y}_{r}\right)
$$

From (5) and (6), we can know the control law that minimizes $J$ is $\boldsymbol{U}=\boldsymbol{P}^{-1}\left[\boldsymbol{Y}_{r}-\boldsymbol{F} y(k)-\boldsymbol{H} \Delta u(k-1)\right]$. Let $\boldsymbol{P}_{1}^{\mathrm{T}}(k)$ represent the first line of $\boldsymbol{P}^{-1}$, then the predictive control law can be written as

$$
\begin{aligned}
\Delta u(k)= & \boldsymbol{P}_{1}^{\mathrm{T}}(k)\left[\boldsymbol{Y}_{r}-\boldsymbol{F} y(k)-\boldsymbol{H} \Delta u(k-1)\right] \\
= & P\left(z^{-1}\right) y_{r}(k+N)+\alpha\left(z^{-1}\right) y(k)+\beta\left(z^{-1}\right) \Delta u(k-1) \\
= & \boldsymbol{Z}_{u}(k) \boldsymbol{\theta}_{u}(k) \\
& \quad u(k)=u(k-1)+\Delta u(k)
\end{aligned}
$$


When designing the model predictive control system, the model predictive control system can not be changed. However, the external interference factors are changeable and complex, and many of them are unpredictable. Therefore, the model prediction system has strong robustness, anti-interference and good practicability. The optimization process of stochastic systems is shown in Figure 2.

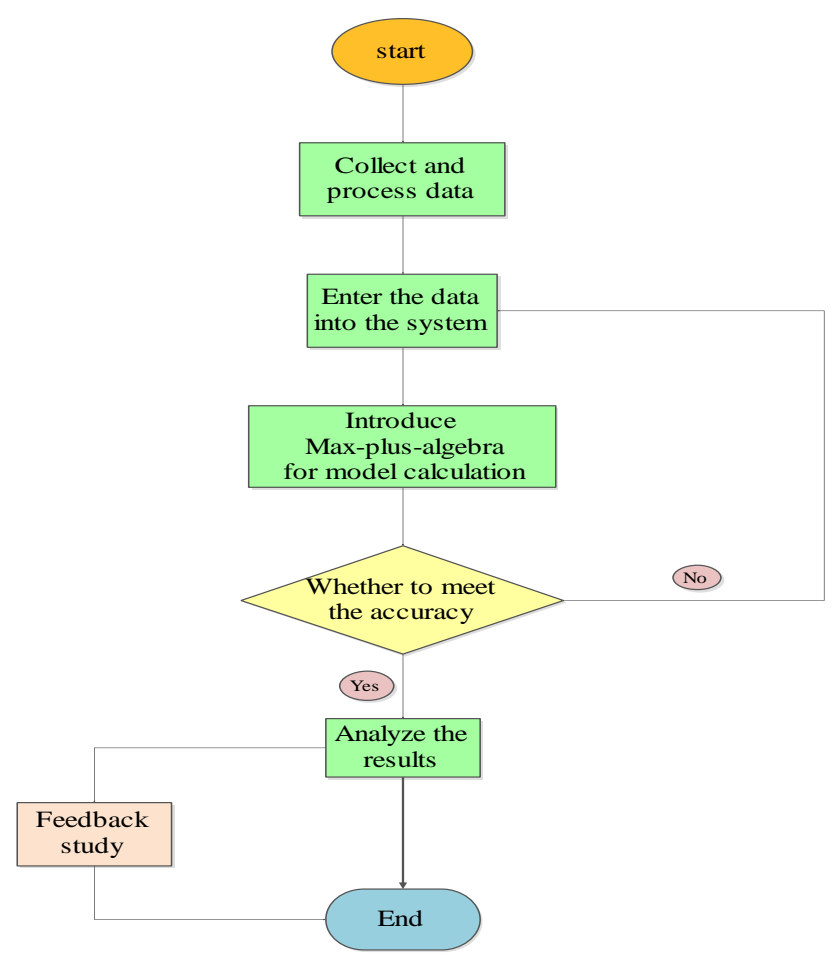

Figure 2. Optimization process of stochastic systems

By introducing the max plus algebra theory and considering the best and worst case fitness, if the model predictive control system can operate normally under two extreme conditions, then when the external conditions are between the two extreme conditions, the model predictive control system can operate normally. The specific operation steps of the model are as follows.

STEP1. Start.

STEP2. Collects and processes data to make data more characteristic.

STEP3. Inputs the processed data into the model predictive control system.

STEP4. Uses the theory of max-plus algebra to operate for the predict control system.

STEP5. Compares the results of the predictive control system with the actual results to see if the accuracy requirements are met. If not satisfied, run the result back to STEP3. Continue with the next step.
STEP6. processes and analyzes the results of the predicts control system, and studies the overall characteristics of the system through the feedback of the operation data.

\section{STEP 7 End.}

\section{Stability AND CONVERGENCE ANALYsis}

Suppose that there exists the optimum control increment $\Delta u^{*}(k-N)=\boldsymbol{S}\left(\boldsymbol{Z}_{u}(k-N)\right) \boldsymbol{\theta}_{u}^{*}$, at time $k$, if the System (1) meets the Conditions A1), A2), the predictive controller is

$$
\Delta u(k)=\boldsymbol{S}\left(\boldsymbol{Z}_{u}(k)\right) \boldsymbol{\theta}_{u}(k)
$$

and the adaptive adjustment law of the parameter vector $\boldsymbol{\theta}_{u}(k)$ is

$$
\boldsymbol{\theta}_{u}(k)= \begin{cases}\hat{\varphi}(k) & |\hat{\varphi}(k)| \leq M_{u} \\ P\{\hat{\varphi}(k)\} & |\hat{\varphi}(k)|>M_{u}\end{cases}
$$

when $0<\gamma \leq 2 / k_{\max }, \lim _{k \rightarrow \infty}|e(k)|=0$.

According to the mean value theorem, we can obtain

$$
\begin{aligned}
& y(k)=f(y(k-1), \cdots, y(k-m), u(k-1) \\
& \left., u(k-2), \cdots,\left(u(k-N-1)+\Delta u^{*}(k-N)\right), \cdots, u(k-n)\right) \\
& =f(y(k-1), \cdots, y(k-m), u(k-1), \\
& \left.u(k-2), \cdots, u(k-N-1)+\boldsymbol{S}\left(\boldsymbol{Z}_{u}(k-N)\right) \boldsymbol{\theta}_{u}(k), \cdots, u(k-n)\right) \\
& y_{r}(k)=f(y(k-1), \cdots, y(k-m), u(k-1) \\
& \left., u(k-2), \cdots,\left(u(k-N-1)+\Delta u^{*}(k-N)\right), \cdots, u(k-n)\right) \\
& =f(y(k-1), \cdots, y(k-m), u(k-1), \\
& \left.u(k-2), \cdots, u(k-N-1)+\boldsymbol{S}\left(\boldsymbol{Z}_{u}(k-N)\right) \boldsymbol{\theta}_{u}^{*}, \cdots, u(k-n)\right)
\end{aligned}
$$

Then, the formula (11) can be expressed as follows.

$$
\begin{aligned}
& e(k)=y(k)-y_{r}(k) \\
& =f(y(k-1), \cdots, y(k-m), u(k-1) \\
& \left., u(k-2), \cdots,\left(u(k-N-1)+\boldsymbol{S}\left(\boldsymbol{Z}_{u}(k-N)\right) \boldsymbol{\theta}_{u}(k)\right), \cdots, u(k-n)\right)- \\
& f(y(k-1), \cdots, y(k-m), u(k-1), \\
& \left.u(k-2), \cdots, u(k-N-1)+\boldsymbol{S}\left(\boldsymbol{Z}_{u}(k-N)\right) \boldsymbol{\theta}_{u}^{*}, \cdots, u(k-n)\right) \\
& \left.=\eta \boldsymbol{S}\left(\boldsymbol{Z}_{u}(k-N)\right) \phi(k-N)\right)
\end{aligned}
$$

In the equation $\phi(k)=\theta_{u}(k)-\theta_{u}^{*}, \eta=\partial f /\left.\partial u(k-N)\right|_{\xi_{u}}, \quad \xi_{u}$ is between $u(k-N-1)+\Delta u^{*}(k-N)$ and $u(k-N)$. From 
the Condition A2 in the original system, it can be known $0<\eta \leq k_{\max }$.

When $V(k)=\phi^{\mathrm{T}}(k) \phi(k)=\|\phi(k)\|^{2}$, it can be obtained as follows.

$$
\begin{aligned}
& V(k)-V(k-N)=\|\phi(k)\|^{2}-\|\phi(k-N)\|^{2} \\
& =\left\|\phi(k-N)-\frac{\gamma \boldsymbol{S}^{\mathrm{T}}\left(\boldsymbol{Z}_{u}(k-N)\right) e(k)}{1+\boldsymbol{S}\left(\boldsymbol{Z}_{u}(k-N)\right) \boldsymbol{S}^{\mathrm{T}}\left(\boldsymbol{Z}_{u}(k-N)\right)}\right\|^{2} \\
& -\|\phi(k-N)\|^{2} \\
& =\frac{\gamma^{2} \boldsymbol{S}\left(\boldsymbol{Z}_{u}(k-N)\right) \boldsymbol{S}^{\mathrm{T}}\left(\boldsymbol{Z}_{u}(k-N)\right) e^{2}(k)}{\left[1+\boldsymbol{S}\left(\boldsymbol{Z}_{u}(k-N)\right) \boldsymbol{S}^{\mathrm{T}}\left(\boldsymbol{Z}_{u}(k-N)\right)\right]^{2}} \\
& -2 \frac{\gamma e(k) \boldsymbol{S}\left(\boldsymbol{Z}_{u}(k-N)\right) \phi(k-N)}{\left[1+\boldsymbol{S}\left(\boldsymbol{Z}_{u}(k-N)\right) \boldsymbol{S}^{\mathrm{T}}\left(\boldsymbol{Z}_{u}(k-N)\right)\right]}
\end{aligned}
$$

That is,

$$
\begin{aligned}
& V(k)-V(k-N)= \\
& \frac{\gamma^{2} \boldsymbol{S}\left(\boldsymbol{Z}_{u}(k-N)\right) \boldsymbol{S}^{\mathrm{T}}\left(\boldsymbol{Z}_{u}(k-N)\right) e^{2}(k)}{\left[1+\boldsymbol{S}\left(\boldsymbol{Z}_{u}(k-N)\right) \boldsymbol{S}^{\mathrm{T}}\left(\boldsymbol{Z}_{u}(k-N)\right)\right]^{2}} \\
& -2 \frac{\gamma e^{2}(k)}{\eta\left[1+\boldsymbol{S}\left(\boldsymbol{Z}_{u}(k-N)\right) \boldsymbol{S}^{\mathrm{T}}\left(\boldsymbol{Z}_{u}(k-N)\right)\right]} \\
& \leq \frac{\gamma^{2} e^{2}(k)}{\left[1+\boldsymbol{S}\left(\boldsymbol{Z}_{u}(k-N)\right) \boldsymbol{S}^{\mathrm{T}}\left(\boldsymbol{Z}_{u}(k-N)\right)\right]} \\
& -2 \frac{\gamma e^{2}(k)}{\eta\left[1+\boldsymbol{S}\left(\boldsymbol{Z}_{u}(k-N)\right) \boldsymbol{S}^{\mathrm{T}}\left(\boldsymbol{Z}_{u}(k-N)\right)\right]} \\
& =\frac{\gamma^{2} e^{2}(k)}{\left[1+\boldsymbol{S}\left(\boldsymbol{Z}_{u}(k-N)\right) \boldsymbol{S}^{\mathrm{T}}\left(\boldsymbol{Z}_{u}(k)\right)\right]}\left[\frac{-2}{\gamma \eta}+1\right] \\
& \text { When }\left\|\boldsymbol{\theta}_{u}(k)-\boldsymbol{\theta}_{u}^{*}\right\|^{2}< \\
& \left\|\boldsymbol{\theta}_{u}(k-N)-\frac{\gamma \boldsymbol{S}\left(\boldsymbol{Z}_{u}(k-N)\right) \boldsymbol{\theta}_{u}(k) e(k)}{1+\boldsymbol{S}\left(\boldsymbol{Z}_{u}(k-N)\right) \boldsymbol{S}^{\mathrm{T}}\left(\boldsymbol{Z}_{u}(k-N)\right)}-\theta_{u}\right\|^{2}
\end{aligned}
$$

it can be obtained as follows.

$$
\begin{aligned}
& V(k)-V(k-N) \\
& <\left\|\boldsymbol{\theta}_{u}(k-N)-\frac{\gamma \boldsymbol{S}\left(\boldsymbol{Z}_{u}(k-N)\right) e(k)}{1+\boldsymbol{S}\left(\boldsymbol{Z}_{u}(k-N)\right) \boldsymbol{S}^{\mathrm{T}}\left(\boldsymbol{Z}_{u}(k-N)\right)}-\theta_{u}^{\prime *}\right\|^{2} \\
& -\left\|\boldsymbol{\theta}_{u}(k-N)-\boldsymbol{\theta}_{u}^{*}\right\|^{2}=\|\phi(k)\|^{2}-\|\phi(k-N)\|^{2}
\end{aligned}
$$

That is,

$$
\begin{gathered}
V(k)-V(k-N) \\
<\frac{\gamma^{2} e^{2}(k)}{\left[1+\boldsymbol{S}\left(\boldsymbol{Z}_{u}(k-N)\right) \boldsymbol{S}^{\mathrm{T}}\left(\boldsymbol{Z}_{u}(k-N)\right)\right]}\left[\frac{-2}{\gamma \eta}+1\right] \\
V(k)-V(k-N) \\
<\frac{\gamma^{2} e^{2}(k)}{\left[1+\boldsymbol{S}\left(\boldsymbol{Z}_{u}(k-N)\right) \boldsymbol{S}^{\mathrm{T}}\left(\boldsymbol{Z}_{u}(k-N)\right)\right]\left[\frac{-2}{\gamma \eta}+1\right]}
\end{gathered}
$$

Suppose

$$
\begin{array}{r}
S=\frac{\gamma^{2} e^{2}(k)}{\left[1+\boldsymbol{S}\left(\boldsymbol{Z}_{u}(k-N)\right) \boldsymbol{S}^{\mathrm{T}}\left(\boldsymbol{Z}_{u}(k-N)\right)\right]} \\
\omega=\left[\frac{-2}{\gamma \eta}+1\right]
\end{array}
$$

Then:

$$
V(k)-V(k-N)<S \omega
$$

When $k$ takes from 1 to $l$ :

$$
\begin{gathered}
V(1)-V(1-N)<S \omega \\
V(2)-V(2-N)<S \omega \\
\vdots \\
V(l)-V(l-N)<S \omega
\end{gathered}
$$

Adding above equations, eliminating $V(1) 、 V(2) \cdots V(l-N)$ term results

$$
0<\sum_{k=l-N+1}^{l} V(k)<\omega \sum_{k=1}^{l} S+\sum_{k=1}^{N} V(k-N)
$$

After taking the limit of the above equation, we can obtain

$$
\omega \lim _{l \rightarrow \infty} \sum_{k=1}^{l} S>-\sum_{k=1}^{N} V(k-N)
$$


For $0<\eta \leq k_{\max }, \quad 0<\gamma \leq \frac{2}{k_{\max }}, \quad \omega=\left[\frac{-2}{\gamma \eta}+1\right] \leq 0$,

$\lim _{l \rightarrow \infty} \sum_{k=1}^{l} S<\left(1-\frac{2}{\gamma \eta}\right)^{-1} \sum_{k=1}^{N} V(k-N)$

Among them, $V(-N+1), \cdots, V(0)$ are determined by the initial value and are bounded quantities,

$$
\lim _{l \rightarrow \infty} \sum_{k=1}^{l} S<\left(1-\frac{2}{\gamma \eta}\right) \sum_{k=1}^{N} V(k-N)<\infty
$$

According to the nature that its general term tends to zero when the series converges, we can obtain

$$
\lim _{k \rightarrow \infty} \frac{\gamma^{2} e^{2}(k)}{\left[1+\boldsymbol{S}\left(\boldsymbol{Z}_{u}(k-N)\right) \boldsymbol{S}^{\mathrm{T}}\left(\boldsymbol{Z}_{u}(k-N)\right)\right]}=0
$$

From the Hypothesis A2) that every partial derivative of $f(\cdot)$ is bounded, we know the input $u(k)$ and output $y(k)$ of the system are bounded, and expect $y_{r}(k)$ is bounded, so $1+\boldsymbol{S}\left(\boldsymbol{Z}_{u}(k-N)\right) \boldsymbol{S}^{\mathrm{T}}\left(\boldsymbol{Z}_{u}(k-N)\right)$ is a bounded quantity, and $\lim _{k \rightarrow \infty}|e(k)|=0$.

\section{Simulation}

Consider the following nonlinear system

$$
\begin{aligned}
& y(k)=2.5 y(k-1) y(k-2) /\left[1+y^{2}(k-1)+\right. \\
& \left.y^{2}(k-2)\right]+1.2 u(k-1)+1.4 u(k-2)+0.7 \\
& \sin (0.5(y(k-1)+y(k-2))) \cos (0.5(y(k-1) \\
& +y(k-2)))
\end{aligned}
$$

Reference sequence $y_{r}(k)$ takes the form of $2+\sin (k \pi T / 20), T=0.01, \quad N=2, \quad N_{u}=1, \quad W_{1}=50 ;$ adaptive ratio $\gamma=0.001$, each component of the initial values of parameter vector $\boldsymbol{\theta}_{u}(-1)$ and $\boldsymbol{\theta}_{u}(0)$ all takes zero.

After data analysis and processing, the following image is obtained.

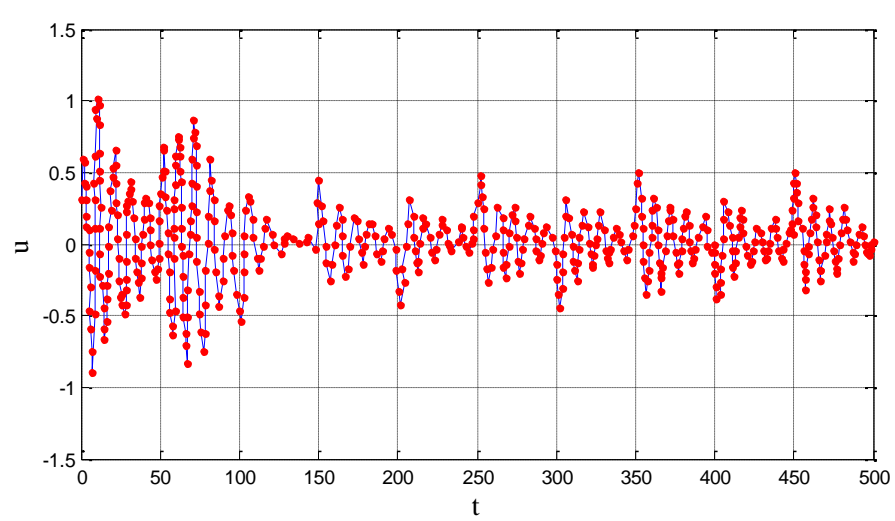

Figure 3. The input curve of stochastic system without optimization

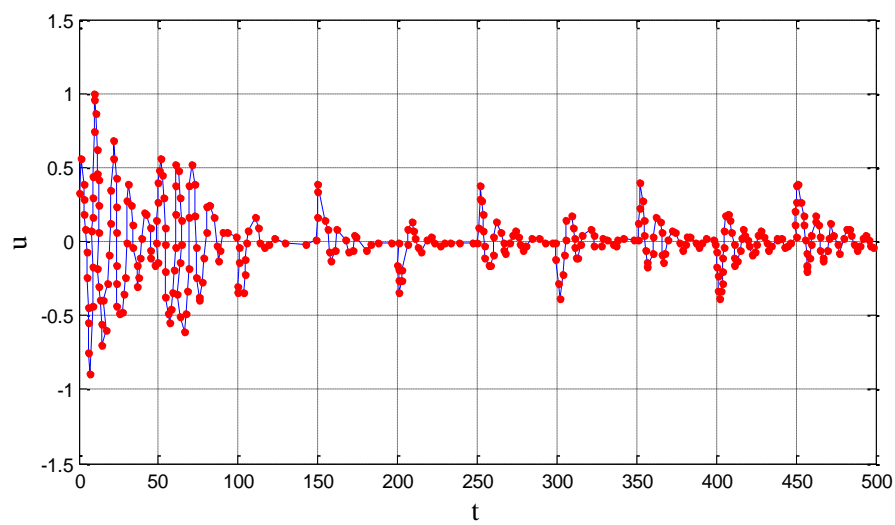

Figure 4. The input curve of stochastic system with optimization

By comparing the system input curves of Fig. 3 and Fig. 4, it is found that the input of the predictive control system is more stable, and the input data is less noisy and less volatile.

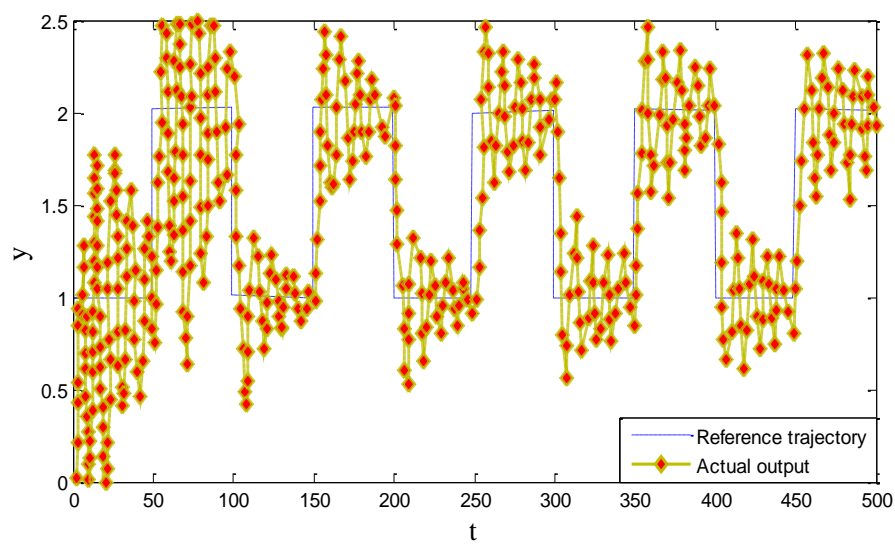

Figure 5. The output curve of stochastic system without optimization 


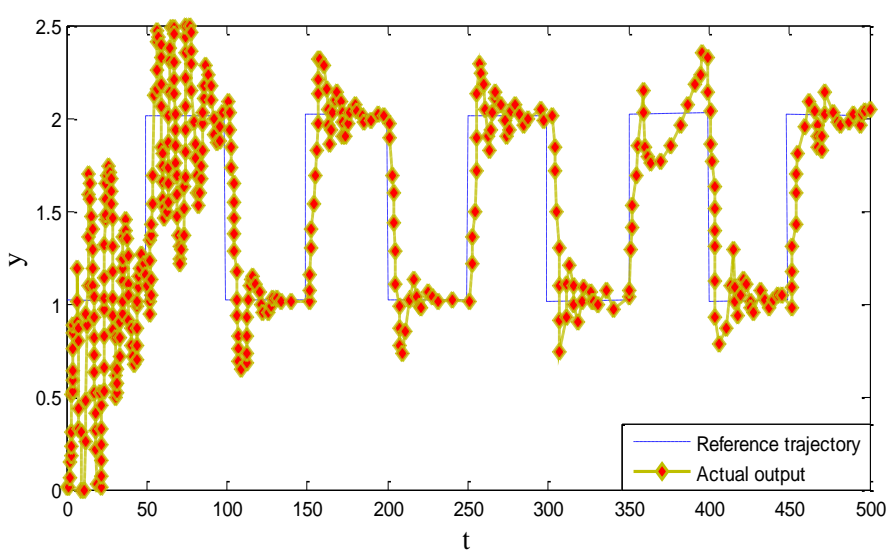

Figure 6. The output curve of stochastic system with optimization

From the simulation results of Fig. 5 and Fig. 6, it can be observed clearly that the output fluctuation of system is small and the accuracy is high compared with the disturbance system.

\section{CONCLUSION}

Compared with traditional algorithm, the method avoids solving the Diophantine equation and matrix inversion. The method need not utilize the unknown constant $g_{0}$. Currently the generalized predictive control has carried out application tests in the field of chemical industry, aerospace, automobile etc., this paper has further improved the timeliness of the predictive control algorithm, and therefore paved the way for the practical application of the algorithm.

\section{ACKNOWLEDGMENT}

The work is supportted by the Science and Technology Innovation Development Program of Jilin City (Grant No. 201831769, 2019301176).

\section{REFERENCES}

[1] H. Genceli, and Nikolaou, M.,Design of Robust Constrained Model Predictive Controllers with Volterra Series, AIChE Journal, Vol.41, No. 9,1995, pp.2098-2107.

[2] W. H. Chen, D. J. Balance, and P. J. Gawthrop,Optimal Control of Nonlinear System: a Predictive Control Approach, Automatica, Vol.39, No. 4, 2003, pp.633-641.

[3] Tanner H G, Loizou S G, and Kyriakopoulos K J.,Nonholonomic navigation and control of cooperating mobile manipulators, IEEE Trans Robotic Autom, Vol.19, No. 1, 2003, pp.53-64.

[4] Barambones O, and Etxebarria V.,Robust neural control forrobotic manipulators,Automatica, Vol.38, No. 2,2002,pp.235-242.

[5] Ho H F, Wong Y K, and Rad A B.,Robust fuzzy tracking con-trol for robotic manipulators,Simulation Modelling Practice and Theory, Vol.4, No. 15,2007, pp.801-816.

[6] Amir Hooshang Mazinan, and Nasser Sadati,Fuzzy predictive control based multiple models strategy for a tubular heat exchanger system,Applied Intelligence, Vol.33, No. 3,2009,pp. 217-263.
[7] Labiod S, Boucheit M S, and Guerrra T M.,Adaptive fuzzy control of a class of MIMO nonlinear systems, Fuzzy Sets and Systems, Vol.15, No. 1,2005,pp.59-77.

[8] Alam M S,and Tokhi M O.Hybird.,fuzzy logic control with genetic optimisation for a single-link flexible manipulator, Engineering Applications of Artificial Intelligence, Vol.6, No. 21,2008, pp.858-873.

\section{Creative Commons Attribution License 4.0 (Attribution 4.0 International, CC BY 4.0)}

This article is published under the terms of the Creative Commons Attribution License 4.0 https://creativecommons.org/licenses/by/4.0/deed.en_US 